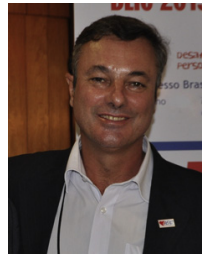

\section{LEFT MAIN CORONARY ARTERY STENOSIS: EVIDENCE AND PATHOPHYSIOLOGY}

\section{To the Editor:}

The commentary by Gaudino and colleagues, ${ }^{1}$ discussing the meta-analysis reported by Gallo and colleagues, ${ }^{2}$ is timely and relevant. Gallo and colleagues ${ }^{2}$ argued that left main coronary artery stenosis (LMCAS) might be considered a separate entity within the coronary artery disease (CAD) spectrum. Apart from the evidence, the explanation for the outcomes in patients with LMCAS can be found in the pathophysiologic understanding of the $\mathrm{CAD}$, which has long been overlooked and has now been greatly reinforced by recent findings. 3

A long-standing view of ischemic heart disease has been the stenosis-centered dogma, in which plaques grow relentlessly in coronaries until they block blood flow, eliciting acute coronary syndrome. Recent evidence has laid waste to this understanding, with the concept that flow-limiting stenosed coronary arteries will lead to so-called chronic myocardial ischemia (CMI), thought to be the causal factor of death. ${ }^{3-6}$ This has thwarted the myth of chronic ischemia in determining the prognosis of patients with CAD. Thus, the acute coronary event does not result mostly from occlusion at the site of severe stenosis seen on a conventional angiogram, as in LMCAS. Instead, the event will be caused by rupture or erosion of a coronary atherosclerotic plaque, most frequently a mild to moderate stenosis or a thin cap fibroatheroma with superimposed thrombus formation, commonly located away from the stable plaque. ${ }^{7,8}$

Therefore, the main mechanism of death for patients with $\mathrm{CAD}$ is no longer ascribed to the stable plaque (left main stenosis) but, rather, to myocardial infarction (MI). CMI appears to be more of a marker for the atherosclerotic burden, such that advanced atherosclerotic CAD carries an increased risk of MI and death because severe lesions have been associated with a larger number of unstable plaques in the coronary tree. The tighter the coronary stenosis, the larger the number of unstable plaques scattered upstream or downstream from the stenotic site, with the potential to rupture and cause MI. ${ }^{7,8}$

\footnotetext{
The Editor welcomes submissions for possible publication in the Letters to the Editor section that consist of commentary on an article published in the Journal or other relevant issues. Authors should: $\bullet$ Include no more than 500 words of text, three authors, and five references. • Type with double-spacing. • See http://jtcs.ctsnetjournals.org/ misc/ifora.shtml for detailed submission instructions. • Submit the letter electronically via jtcvs.editorialmanager.com. Letters commenting on an article published in the JTCVS will be considered if they are received within 6 weeks of the time the article was published. Authors of the article being commented on will be given an opportunity of offer a timely response ( 2 weeks) to the letter. Authors of letters will be notified that the letter has been received. Unpublished letters cannot be returned.
}

The main aim of improving the prognosis of patients with CAD is related to a therapy able to protect against future acute coronary thrombotic events. Coronary artery bypass grafting can address the existing lesion and future culprit lesions; however, percutaneous coronary intervention can only address the stable lesion, leaving the future lesions at risk. ${ }^{9}$

Based on the pathophysiologic concept, the left main (LM) lesion will have no or negligible effects on the outcomes of MI and death. Isolated LM disease was evident in $\sim 15 \%$ of patients, and 3-vessel disease was present in only $20 \%$ of cases. All randomized controlled trials of patients with LMCAS had enrolled only low- and intermediate-risk patients, and their results should not be extrapolated to patients with greater CAD complexity. ${ }^{2}$

With the recent evidence, plus the understanding of the pathophysiologic concepts, a remarkable transformation has been underway and must be incorporated into the guidelines. The 2018 European guidelines on myocardial revascularization still recommended that LM lesion treatment should be based on documented ischemia, a hemodynamically relevant lesion, or $>50 \%$ LM stenosis, a now-defunct definition. ${ }^{10}$

In conclusion, the pathophysiologic principles have reinforced the evidence that LMCAS is not a separate entity but shares the same characteristics as non-LM CAD. Therefore, it is reasonable that it should be managed accordingly.

\section{Walter J. Gomes, $M D, P h D$ Cardiovascular Surgery Discipline Escola Paulista de Medicina and São Paulo Hospital Federal University of São Paulo São Paulo, Brazil}

\section{References}

1. Gaudino M, Pagano D, Freemantle N. Commentary: the left main controversy: is this a real subgroup requiring bespoke clinical recommendations? J Thorac Cardiovasc Surg. May 6, 2020 [Epub ahead of print].

2. Gallo M, Blitzer D, Laforgia PL, Doulamis IP, Perrin N, Bortolussi G, et al. PCI vs. CABG for left main coronary artery disease: a meta-analysis. J Thorac Cardiovasc Surg. April 15, 2020 [Epub ahead of print].

3. Maron DJ, Hochman JS, Reynolds HR, Bangalore S, O'Brien SM, Boden WE, Ischemia Research Group. Initial invasive or conservative strategy for stable coronary disease. N Engl J Med. 2020;382:1395-407.

4. Bangalore S, Maron DJ, O’Brien SM, Fleg JL, Kretov EI, Briguori C, IschemiaCKD Research Group. Management of coronary disease in patients with advanced kidney disease. N Engl J Med. 2020;382:1608-18.

5. Libby P, Nahrendorf M, Swirski FK. Leukocytes link local and systemic inflammation in ischemic cardiovascular disease: an expanded "cardiovascular continuum". J Am Coll Cardiol. 2016;67:1091-103.

6. Rezende PC, Ribas FF, Serrano CV Jr, Hueb W. Clinical significance of chronic myocardial ischemia in coronary artery disease patients. J Thorac Dis. 2019;11: 1005-15.

7. Vancheri F, Longo G, Vancheri S, Danial JSH, Henein MY. Coronary artery microcalcification: imaging and clinical implications. Diagnostics (Basel). 2019;9: 125.

8. Arbab-Zadeh A, Fuster V. The myth of the "vulnerable plaque": transitioning from a focus on individual lesions to atherosclerotic disease burden for coronary artery disease risk assessment. J Am Coll Cardiol. 2015;65:846-55. 
The author reported no conflicts of interest.

The Journal policy requires editors and reviewers to disclose conflicts of interest and to decline handling or reviewing manuscripts for which they may have a conflict of interest. The editors and reviewers of this article have no conflicts of interest.

9. Mancini GB, Hartigan PM, Bates ER, Sedlis SP, Maron DJ, Spertus JA, COURAGE Investigators and Coordinators. Angiographic disease progression and residual risk of cardiovascular events while on optimal medical therapy: observations from the COURAGE Trial. Circ Cardiovasc Interv. 2011;4:545-52.

10. Neumann FJ, Sousa-Uva M, Ahlsson A, Alfonso F, Banning AP, Benedetto U, et al. 2018 ESC/EACTS guidelines on myocardial revascularization. Eur Heart J. 2019;40:87-165.

https://doi.org/10.1016/j.jtcvs.2020.05.102

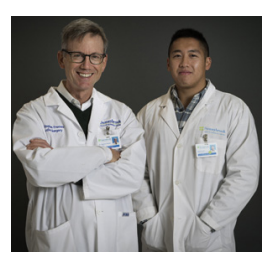

REPLY: GOING FROM STABLE TO UNSTABLE Reply to the Editor:

We thank Dr Gomes for his letter to the editor and his nuanced discussion on the pathophysiology of complex coronary artery disease (CAD), particularly as it relates to left main stenosis. ${ }^{1}$ This letter, in response to a commentary by Gaudino and colleagues, ${ }^{2}$ further expands on the idea that left main disease should not be considered a separate entity, either from a statistical basis as described by Gaudino and colleagues or from a pathophysiologic perspective as highlighted by Dr Gomes.

The recent publication of the ISCHEMIA trial showed no difference in the primary outcome of a composite of cardiovascular death, myocardial infarction, or heart failure rehospitalization in those with stable CAD and moderate or severe ischemia treated with medical therapy or invasive angiography and revascularization. ${ }^{3}$ Although many of these patients had stable multivessel disease $(31.4 \%$ had double-vessel whereas $45.1 \%$ had triple-vessel disease), patients who had significant left main disease on coronary computed tomography angiography were excluded. ${ }^{4}$ The extent of CAD was associated with both all-cause mortality and myocardial infarction. Furthermore, ISCHEMIA investigators recently presented a subgroup analysis comparing patients with intermediate left main lesions (25\%-49\%) versus those without intermediate left main lesions and found that the incidence of the primary outcome was greater in those with intermediate left main lesions, suggesting that the burden of CAD is also greater in this group. ${ }^{5}$ Early work in the SYNTAX (Synergy Between Percutaneous Coronary Intervention With Taxus and Cardiac Surgery) subgroup of left main patients suggest that lower SYNTAX score left main patients had similar number of major adverse cardiac and cerebrovascular events after percutaneous coronary intervention (PCI) or coronary artery bypass grafting (CABG) whereas major adverse cardiac and cerebrovascular events were much greater in those with high SYNTAX scores after PCI compared with $\mathrm{CABG}{ }^{6}$ The development of the SYNTAX II score suggests that the incorporation of anatomical complexity with patient factors help predict long-term mortality. ${ }^{7}$ All this evidence reinforces the notion that the extent of CAD is prognostically important. As Dr Gomes elegantly points out, those with left main disease may have a greater total burden of CAD throughout the coronary tree that are at risk for plaque rupture leading to acute coronary syndrome (ACS) and death-the incidence of true isolated left main disease is likely low. ${ }^{1}$

Thus, it is not uncommon for patients with left main disease to present as ACS. In the trials comparing CABG with PCI in left main disease, the proportion of patients with ACS varied: in EXCEL (Evaluation of XIENCE versus Coronary Artery Bypass Surgery for Effectiveness of Left Main Revascularization) and PRECOMBAT (Premier of Randomized Comparison of Bypass Surgery versus Angioplasty Using Sirolimus-Eluting Stent in Patients with Left Main Coronary Artery Disease), about $60 \%$ of patients presented with stable angina or silent ischemia whereas the remaining patients presented with a recent myocardial infarction (within 7 days) or unstable angina. The numbers were lower in the NOBLE (Nordic-Baltic-British left main revascularisation study), only $18 \%$ of patients presented with ACS whereas the remaining had stable angina. Overall, the majority of randomized evidence comparing PCI with $\mathrm{CABG}$ in left main patients was derived predominantly from patients with stable CAD. Although many guidelines indicate that stable ischemic heart disease and ACS should be managed similarly, PCI, in general may be more appropriate than $\mathrm{CABG}$ for most patients. ${ }^{8,9}$ However, in those with left main and multivessel CAD, CABG should be the preferred treatment for both patients with stable CAD and ACS.

The evidence landscape around coronary revascularization is rapidly evolving, yet we are tasked with optimally managing the patients in front us. The management of these patients in the face of uncertainty highlights the evergrowing importance of the heart team. While CABG should be the preferred strategy in those who are surgical candidates, the personalization and tailoring of treatment strategies for those who are less than ideal for surgery in a heart team discussion can help optimize these decisions. ${ }^{10}$

Derrick Y. Tam, $M D, P h D$

Stephen E. Fremes, $M D, M S c$

Division of Cardiac Surgery

Schulich Heart Centre

Department of Surgery

Sunnybrook Health Sciences Centre 